THE DEPTH OF CONVECTION IN A FLUID WITH STRONGLY TEMPERATURE AND PRESSURE DEPENDENT VISCOSITY

\title{
A.C. Fowler
}

Department of Mathematics, Massachusetts Institute of Technology, Cambridge, MA 02139

Abstract. We discuss the equations describing convective motion of a fluid with temperature and pressure dependent rheology. By combining observational constraints from plate tectonics with theoretical constraints deduced from the model equations and parameter values, we are led to the conclusion that shallow upper mantle convection is consistent with the equations of fluid dynamics.

\section{The Nature of Mantle Convection}

In studying models of the convective flow in the earth's mantle (for reviews, see Oxburgh and Turcotte (1978), Turcotte (1979)), geophysicists have been hampered by an unusual set of circumstances: these are, that direct observational evidence is sketchy and requires interpretation; direct laboratory simulation appears insurmountably difficult (due to a lack of suitable materials, and to the inability to scale all the parameters properly); direct numerical computation of even a two-dimensional convective flow with a relevant rheology has not been obtained. These circumstances have produced a situation in which various fundamental properties of the convective flow are not well understood; for example, the depth of convection is still a contentious issue, with various authors arguing for whole-mantle or layered upper and lower mantle convection, respectively. Because theoretical and numerical studies of the convection of a strongly nonlinearly rheologic fluid are effectively lacking (in the context of the earth), there is a tendency to make deductions on the nature of the real flow from much simpler (often, for example, Newtonian) models in the hope that they are accurate enough (in some sense).

of the various complications associated with mantle convection, possibly the most obvious and perhaps the most significant is the dependence of viscosity on temperature and pressure. Commonly quoted activation energies for mantle-1ike materials are $E^{*}=122 \mathrm{kcal} / \mathrm{mole}$ (Goetze 1978), and with $a$ basal temperature of (say) $\mathrm{T}_{b}=2000 \mathrm{~K}$, one finds the dimensionless parameter which measures the strength of viscosity dependence on temperature, $\mathrm{E} * / \mathrm{RT}_{\mathrm{b}} \sim 30$. The pressure dependence is of a similar order of magnitude, and operates in the opposite direction. Even if the activation energy were much less, $E * / \mathrm{RT}_{\mathrm{b}}$ would still be large, and it is the fact that it occurs in an exponential dependence that is so cructal. Local variation of the temperature by a few hundred degrees Kelvin will alter the viscosity by orders of magnitude, and this can hardly but affect the flow. We suggest that the rheology

Copyright 1982 by the American Geophysical Union.

Paper number 2L0773.

$0094-8276 / 82 / 002 L-0773 \$ 3.00$ will therefore control the flow, and thus that models which ignore the rheology must be carefully examined to see if conclusions deduced from them will carry over. Particularly we will briefly propose an analogue of Turcotte and Oxburgh's (1967) boundary layer theory, to see if the conclusions of Elsasser, Olson and Marsh (1979) will carry across: we find they do not. (See also O'Connell and Hager 1980 , for a different viewpoint.) The idea of rheology controlling the internal temperature has been developed by Tozer $(1972,1977,1978)$, but we emphasize that his is essentially a global idea, whereas we shall be concerned here with a local dynamic control.

The Effect of a Strongly Temperature and Pressure Dependent Rheology

Let us consider the convection of a fluid whose viscosity is given by

$$
\eta=n_{0} \exp \left[\left(E^{*}+p^{*}\right) / R T\right]
$$

where $\eta_{0}$ is a reference viscosity, $E^{*}$ is the activation energy, $p$ is the pressure, $V *$ is the activation volume, $R$ is the gas constant, and $T$ is the absolute temperature. To $\mathrm{f} i \mathrm{x}$ ideas (only), we consider prescribed temperatures $T_{b}$ on the base, and $T_{0}$ (substantially less then $T_{b}$ ) on the surface. $\mathrm{E}^{*} / \mathrm{RT}_{\mathrm{b}}$ is a dimensionless measure of how much the exponent varies through the flow. If we denote

$$
\varepsilon=R T_{b} / E * \ll 1
$$

then the viscosity contrast between top and bottom is $\exp [0(1 / \varepsilon)]$. If $1 / \varepsilon \sim 30$, then $\exp (1 / \varepsilon)$ $10^{13}$, which is beyond the range of any laboratory or numerical experiment so far conducted. Vertical temperature profiles for which $T /\left(E *+p V^{*}\right)$ are constant are isoviscous: for a single phase mantle in which pressure is essentially lithostatic, such isoviscous temperatures are linear functions of depth.

Now suppose the maximum value of $T /\left(E^{*}+p^{*}\right)$ within a convection cell gives a corresponding minimum viscosity $\eta_{\mathrm{m}}$. If the temperature elsewhere is much smaller than the corresponding isoviscous profile (with $\eta=\eta_{m}$ ), then it follows from the smallness of $\varepsilon$ that the viscosity in such regions will be exponentially large in comparison with $\eta_{m}$. From the point of view of the minimum viscosity material, the high viscosity material will appear effectively rigid. Note: we do not mean rigid in the sense of behaving elastically rather than viscously, but dynamically rigid in the sense that such high viscosity material will appear rigid on time scales relevant to the convection. Since an applied stress induces the largest velocity in the least viscous 
fluid, we might expect the convective time scale to be dictated by such fluid.

Effectively rigid material will then not significantly deform on time scales of interest, and it seems unlikely that it can participate substantially in the circulatory motion: a little thought suggests that one plausible state will be that 'cold', 'rigid' material will exist in one or more quasi-stagnant layers throughout the cell, their location depending on the relation of the isoviscous temperature profiles to the interior (adiabatic) temperature. Particularly, vigorous convection (high Rayleigh number) will induce a top cold thermal boundary layer, which will be 'quasi-rigid' and preferentially stagnant; this rigid boundary layer may be defined as the 1ithosphere. The location of other such rigid regions depends on the precise rheology, and is discussed further below.

In the real earth, of course, such stagnant plates do not exist, rather the tectonic plates are active, moving relative to each other at speeds of $\delta 10 \mathrm{~cm} y^{-1}$. The reason for such activity is the nature of the motion at oceanic trenches, where the surface plates are subducted downwards into the upper mantle. The discussion above, however, raises the question of how such cold, rigid slabs are able to be subducted at all: various studies (Turcotte et al 1977, Turcotte et al 1978) have shown convincingly that subduction may be understood on the basis of nonhydrodynamic phenomena such as partial melting and elastic yielding, but the problem of initiation remains puzzling.

For a thermoviscous fluid, the growing instability of the cold layer as it thickens is overcompensated by the increasing effective viscosity, and purely buoyant effects will not subduct the lithosphere (Yuen, Peltier and Schubert 1981) - non-hydrodynamic processes must be relevant. on the other hand, the decrease in viscosity in a basal thermal layer will greatly enhance instability. Calculations of Schubert et al (1969) can be adapted (Fowler, 1981) to show that a temperature difference across the layer greater than $O\left(\varepsilon T_{b}\right)$ will be catastrophically unstable, and hence in a steady state such a difference will not occur. In effect, the basal thermal boundary layer vanishes, and the temperature and viscosity are (approximately) continuous at the base. This is really just another instance of the isoviscous nature of the flow.

\section{Constraints on the Viscosity}

Consider the convection of a fluid in a layer of depth $d$. We nondimensionalise the temperature $T$ and depth $y$ by putting

$$
\mathrm{T}=\mathrm{T}_{\mathrm{b}} \theta, \quad \mathrm{y}=\mathrm{dy} \mathrm{y}^{*}
$$

where $\mathrm{T}_{\mathrm{b}}$ is the basal temperature. If $\rho_{0}$ is the ambient density (with the Boussinesq approximation), $g$ is the constant acceleration due to gravity, then the (1ithostatic) pressure is given by

$$
p=\rho_{0} g d(1-y),
$$

where henceforward we drop the asterisk on $y^{*}$, $y=1$ is the top surface, and $y=0$ is the base. We define the basal viscosity $n_{m}$ by

$$
\eta_{m}=n_{0} \exp \left[\left(E^{*}+\rho_{0} g d V *\right) / R T_{b}\right],
$$

where $E^{*}$ is activation energy, $V^{*}$ is activation volume, $R$ is the gas constant; it then follows from (1) that

$$
n=\eta_{m} \exp [\{(1+\mu)(1-\theta)-\mu y\} / \varepsilon \theta],
$$

where

$$
\varepsilon=\mathrm{RT}_{\mathrm{b}} / \mathrm{E}^{*}, \quad \mu=\rho_{0} \mathrm{gdV} * / \mathrm{E}^{*} .
$$

With values $\rho_{0} \sim 3.5 \mathrm{gm} \mathrm{cm}-3, g \sim 10 \mathrm{~m} \mathrm{~s}^{-2}, \mathrm{~d} \sim$ $700 \mathrm{~km}, \mathrm{~V}^{*} \sim 11 \mathrm{~cm}^{3} \mathrm{~mole}^{-1}, \mathrm{E} * \sim 122 \mathrm{kcal} \mathrm{mole}^{-1}$, $\mathrm{T}_{\mathrm{b}} \sim 2000 \mathrm{~K}, \mathrm{R} \sim 8.3 \mathrm{~J} \mathrm{~mole}^{-1} \mathrm{~K}^{-1}$, we have

$$
\varepsilon \sim 1 / 30, \mu \sim 1 / 2 .
$$

These parameters are for shallow mantle convection, but for illustration only, to show that $\varepsilon \ll 1, \mu \sim 1$.

In terms of $\theta$, we have proposed that a basal boundary layer will have a temperature jump $\Delta \theta \sim O(\varepsilon)$. (6) then implies that $n$ varies by $\lesssim O(1)$ within the layer, and so the isoviscous temperature $\theta_{\text {rhe }}$ which one would expect in the cell interior is given by

$$
\theta=\theta_{\text {rhe }} \equiv 1-\mu y /(1+\mu)+O(\varepsilon) \text {. }
$$

We reiterate this point: if $(1+\mu)(1-\theta)-\mu y$ becomes positive and $O(1)$ in the cell interior, i.e. $\theta_{\text {rhe }}-\theta \sim 0(1)$, then $n / n_{m} \sim \exp [0(1 / \varepsilon)]$, and such material is effectively rigid (corresponding considerations apply if $\theta-\theta$ rhe $\sim 0(1))$. Therefore we expect that convection in a fluid with such a rheology will constrain the interior temperature profile to be approximately isoviscous. This is in line with models of the earth's viscosity which are consistent with isostatic rebound data (Cathles 1975, Peltier 1980). However, the 'isoviscous' nature of the flow is paradoxically due to the strongly temperaturedependent rheology, and it remains to be seen what other effects the rheology will have.

The above discussion raises an immediate problem. If the rheology chooses the temperature, what does the temperature equation do? There does not seem to be any possible way in which it does not also choose the temperature; particularly, for a vigorously convecting fluid at high Rayleigh number, the appropriately scaled temperature equation will be of singular perturbation type, and in the absence of viscous or radiogenic source terms, we can expect that thermal boundary layers will occur, but that elsewhere (i.e., in the interior) the entropy will be nearly constant, which in the present context implies an internal adiabatic temperature. In view of the small temperature jump across the basal layer, we may then write

$$
\theta \approx \theta_{\text {ad }}=\exp [-D y],
$$

where

$$
D=\alpha g d / c_{p}
$$


is the dissipation number, $\alpha$ is the thermal expansion coefficient, $c_{p}$ is the specific heat. For shallow mantle convection, $D \sim 0.2$; for whole mantle convection, $D \sim 0.86$. We now have two proposed temperatures for the sublithospheric flow; furthermore, these are formally distinct (one is exponential with depth, the other linear), and therefore not in principle reconcilable.

This is a puzzle, and apparently the solution is the following. We do not require $\theta$ ad to equal

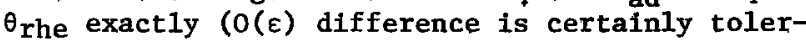
ated): what we formally require is that $\left|\theta_{\text {ad }}-\theta_{\text {rhe }}\right| \ll 1$, and this can be satisfied provided the parameters $D$ and $\mu$ are appropriately prescribed. Specifically, we require formally that $\mathrm{D} \ll 1$, so that $\theta_{\mathrm{ad}} \approx 1-\mathrm{Dy}+1_{2} \mathrm{D}^{2} \mathrm{y}^{2}+\ldots$, and then also that $D-\mu /(1+\mu) \ll 1$, so that $\left|\theta_{\text {ad }}-\theta_{\text {rhe }}\right|=0\left[|D-\mu /(1+\mu)|, D^{2}\right]$. This requires (if $\left|\theta_{\text {ad }}-\theta_{\text {rhe }}\right| \sim O(\varepsilon)$ ) that

$$
D-\mu /(1+\mu) \sim D^{2} \sim O(\varepsilon) .
$$

It is evident that shallow mantle convection is reasonably consistent with (12), whereas whole mantle convection is definitely not. How can 'given' parameters be prescribed? Examining D and $\mu$, we see that each is proportional to depth $d$, and therefore (12) can always be satisfied provided the depth is sufficiently small. This implies, since the depth is an externally prescribed constant, that the depth of vigorous convection (limited by (12)) may be smaller than the layer depth. From previous discussion, we might expect the remalnder of the layer to consist of more viscous, quasi-stagnant fluid. Such behavior is suggested by various numerical computations (for example Torrance and Turcotte (1971)).

The constraint (12) suggests that even if one attempted to achieve whole mantle convection, the natural convecting depth would be shallow, and the lower mantle would behave effectively stagnantly, or convect separately. A more detailed scaling analysis (Fowler, 1981) suggests that the upper mantle convection will admit an algebraic variation in viscosity (of perhaps 2 to 4 orders of magnitude), and this is in line with a numerical simulation of Parmentier and Turcotte (1978). The constraints (12) are replaced by

$$
D \ll 1, \mu-(1+\mu) D=m \in \ln (D / E),
$$

where for a stress free base, $m \approx 2$, for a no slip base $m \approx 5$. It is perhaps revealing that with $\mu \sim 0.5, D \sim 0.2, \varepsilon \sim 1 / 30$, we find $m \approx 3.35$ from (13), whereas for whole mantle convection with $\mathrm{D} \sim 0.86, \mu \sim 2$, (13) would imply $\mathrm{m}<0$. We should emphasize that it is the orders of magnitude which are important here, not the precise quantitites. The present discussion is therefore fairly robust under variation of material properties such as activation energy, etc.

\section{Discussion}

What we have attempted to show is that purely mechanical processes will have the effect of preferentially selecting a shallow depth of vigorous convection. Such a conclusion appears to receive observational support from the compressive nature of deep earthquakes (Isacks and Molnar 1971), and the fact that such earthquakes (identified with the integrity of slabs at such a depth) do not occur below 700 kilometre depth. In addition, a seismic discontinuity at $\sim 670 \mathrm{~km}$ may be due to a phase change and a chemical discontinuity, either of which could act as a barrier to convection. Also, the different nature of ocean island basalts (due to hot spots) and mid-ocean ridge basalts circumstantially suggests a chemically layered mantle (Anderson 1979, 1980, 1981). The apparent coincidence between a chemical discontinuity at $\sim 700 \mathrm{~km}$ acting as a barrier to convection, and the apparently mechanically preferred depth is readily explained in terms of subducting slabs which are able to maintain the chemical layering at depth. For example, if a layer is too deep, slabs preferentially sink to the natural convective depth, thus providing a natural fluid dynamic mechanism whereby a chemically layered mantle could occur.

\section{References}

Anderson, D.L., Chemical stratification of the mantle, J. Geophys. Res. 84, 6297-6298, 1979.

Anderson, D.L., Early evolution of the mantle, Episodes No. 3, 3-7, 1980.

Anderson, D.L., The chemical composition and evolution of the mantle, preprint, 1981.

Cathles, L.M., IIr, The viscosity of the earth's mant1e, Princeton University Press, Princeton, N.J., 1975.

Elsasser, W.H., P. Olson, and B.D. Marsh, The depth of mantle convection, J. Geophys. Res. 84, 147-155, 1979.

Fowler, A.C., Implications of scaling and nondimensionalisation for the convection of the earth's mantle, preprint, 1981.

Goetze, C., The mechanics of creep in olivine, Phi1. Trans. Roy. Soc. A 288, 99-119, 1978.

Isacks, B., and P. Molnar, Distribution of stresses in the descending lithosphere from a global survey of focal-mechanism solutions of mantle earthquakes, Rev. Geophys. Space Phys. 9, 103-174, 1971.

0 'Connell, R.J., and B.H. Hager, On the thermal state of the earth, in Physics of the Earth's Interior, 270-317, Soc. Italiana di F1sica, Bologna, Italy, 1980.

Oxburgh, E.R., and D.L. Turcotte, Mechanisms of continental drift, Rep. Prog. Phys. 41, 1249-1312, 1978.

Parmentier, E.M, , and D.L. Turcotte, Two-dimensional mantle flow beneath a rigid accreting 11thosphere, Phys. Earth Planet. Int. 17, 281-289, 1978 .

Peltier, W.R., Mantle convection and viscosity, in Physics of the Earth's Interior, 361-431, Soc. Italiana d1 Fisica, Bologna, Italy, 1980.

Schubert, G., D.L. Turcotte, and E.R. Oxburgh, Stability of planetary interiors, Geophys. J. R. astr. Soc. 18, 441-460, 1969.

Torrance, K.E., and D.L. Turcotte, Thermal convection with large viscosity variations, $\mathrm{J}$. F1uid Mech., 47, 113-125, 1971.

Tozer, D.C., The present thermal state of the terrestrial planets, Phys. Earth Planet. Int. 6, 182-197, 1972.

Tozer, D.C., The thermal state and evolution of 
the earth and terrestrial planets, Sci. Progr. $64,1-28,1977$.

Tozer, D.C., Terrestrial planet evolution and the observational consequences of their formation, in The Origin of the Solar System, ed. S.F. Dermott, Wiley, New York, N.Y., 433-462, 1978.

Turcotte, D.L., Convection, Revs. Geophys. Space Phys. 17, 1090-1098, 1979.

Turcotte, D.L., W.F. Haxby, and J.R. Ockendon, Lithospheric instabilities, in Island Arcs, Deep Sea Trenches, and Back Arc Basins, ed. M. Talwani and W.C. Pitman III, 63-69, A.G.U., Washington, D.C., 1977.

Turcotte, D.L., D.C. McAdoo, and J.G. Caldwe11,
An elastic-perfectly plastic analysis of the bending of the 1ithosphere at a trench, Tectonophys. 47, 193-205, 1978.

Turcotte, D.L., and E.R. Oxburgh, Finite amplitude convective cells and continental drift, J. Fluid Mech. 28, 29-42, 1967.

Yuen, D.A., W.R. Peltier and G. Schubert, on the existence of a second scale of convection in the upper mantle, Geophys. J. R. Astro. Soc. 65, 171-190, 1981 .

(Received September 14, 1981; accepted May 14, 1982.) 